\title{
Molecules in the mirror: how SERS backgrounds arise from the quantum method of images $\dagger$
}

Cite this: Phys. Chem. Chem. Phys., 2014, 16, 6544

Received 8th January 2014 Accepted 20th February 2014

DOI: $10.1039 / c 4 c p 00093 e$

www.rsc.org/pccp

The Raman coupling of light to molecular vibrations is strongly modified when they are placed near a plasmonic metal surface, with the appearance of a strong broad continuum background in addition to the normal surface-enhanced Raman scattering (SERS) peaks. Using a quantum method of images approach, we produce a simple but quantitative explanation of the inevitable presence of the background, due to the resistive damping of the image molecule. This model thus suggests new strategies for enhancing the SERS peak to background ratio.

The coupling of light to molecules is a key aspect of both fundamental and applied spectroscopy, and widely used as the basis of diagnostics, biological sensing, chemical analysis, as well as much of surface science. ${ }^{1-5}$ When molecules are placed on metal surfaces their interaction with light of wavelength $\lambda$ is modified from a variety of effects. Firstly, absorption and emission are suppressed by the null in the optical field at the metal surface caused by interference with the out-of-phase reflection. As the molecule is lifted above the metal surface, however, absorption and emission are enhanced for heights of $\sim \lambda / 4 .^{6}$ Secondly, for certain (notably coinage) metals, plasmonic resonances exist on the surface with a high density of states, into which molecules can emit light very efficiently, so that when molecules approach the surface they feel the enhanced electric field, $E$, produced by these resonances. This has the effect of increasing their emission rates, although once the molecule is closer than $\sim 10 \mathrm{~nm}$, quenching of emission occurs. ${ }^{6}$ Luminescence, Rayleigh and SERS (which is the focus here) are all strongly enhanced by the presence of plasmonic metal surfaces.

SERS is a more than thirty year old technique for enhancing the Raman scattering of molecules. ${ }^{7}$ The recent availability of a variety of nanostructured plasmonic particles and substrates,

\footnotetext{
${ }^{a}$ School of Physics and Astronomy, University of Glasgow, Glasgow G12 $8 Q Q$, UK

${ }^{b}$ Cavendish Laboratory, Department of Physics, University of Cambridge,

Cambridge, CB3OHE, UK. E-mail: jjb12@cam.ac.uk

$\dagger$ Electronic supplementary information (ESI) available: Additional derivation of eqn (5). See DOI: 10.1039/c4cp00093e
}

with large numbers of 'hot-spots', increases the average overall $E$ field and allows dramatic enhancement of the SERS signal. ${ }^{8}$ This has given rise to a huge interest in SERS spectroscopies. Over the years, however, there have been strong debates over the origin of the SERS signals and a variety of mechanisms for this effect have been proposed. ${ }^{9-12}$ While it is well established that the enhancement (scaling as $\left.|E|^{4}\right)^{7}$ is due to the excitation of the plasmonic resonance at the surface, there is discussion of the extent to which the pure electromagnetic enhancement is supplemented by a chemical contribution, and experiments suggest that the electronic structure of the molecules is actually modified in some SERS experiments. ${ }^{1,13,14}$ More puzzling still is that SERS vibrational Raman peaks are always accompanied by a spectrally broad background, which is increased by a similar factor to the SERS itself. It is this increased broad background that interests us here. The origin of this background has been the subject of much debate, and little consensus exists. ${ }^{10,11,15}$ Recently we established that on quantitatively reproducible substrates, (i) the SERS background requires both molecules and plasmons, (ii) is sensitive to the chemical nature of the surface, and (iii) is not seen for the anti-Stokes emission. ${ }^{16}$

Here we use the method of images technique ${ }^{17}$ to develop a new model for the coupling of light to molecules on plasmonicallyactive metal nanostructures which explains the presence of the broad SERS background. ${ }^{17}$ Using this approach the interaction between the molecule and the nearby plasmonic surface (Fig. 1a) is treated as if there were no surface at all, but the molecule is accompanied by an image molecule (Fig. 1b), the properties of which are designed to take account of the required boundary conditions. The two molecules (real and its image) then interact with the exponentially localized surface plasmon resonance to produce the Raman spectrum. The physical picture can be thought of as two oscillators (vibrating at the frequency of their excited molecular vibrational modes, $\nu$ ), coupled by the dipoledipole interaction. The molecular image dipole is strongly damped from its excited vibrational state to its vibrational ground state at a rate, $\Gamma$, by resistive effects inside the metal while the real molecule is damped between these two states at a 


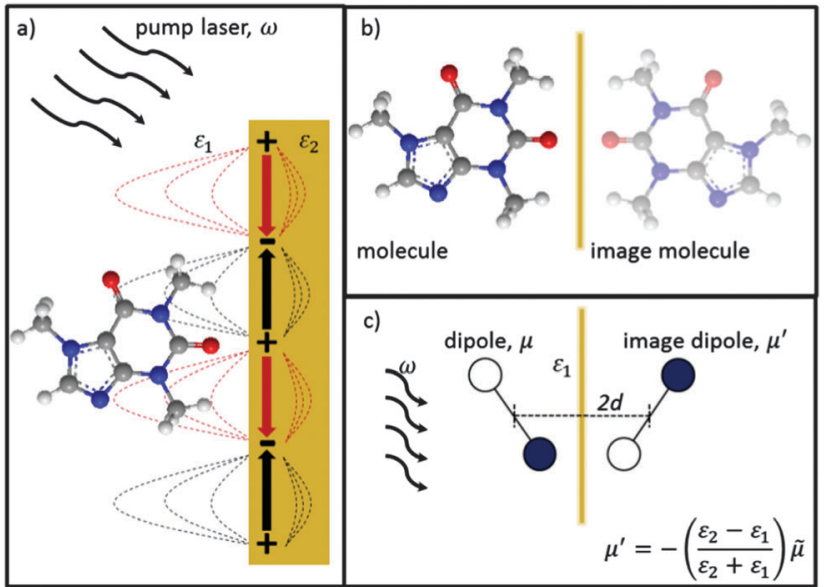

Fig. 1 Representation of method of images approach with (a) real molecule interacting with a plasmonic metal surface replaced by (b) a real-moleculeimage-molecule interaction. (c) Dominant molecular vibrating bond of real and image molecules now represented by dipoles. Real and image dipoles separated by distance, $2 d$, and $\omega$ is the exciting laser field.

rate, $\gamma$, by the metal surface. Note that accounting for the electric field boundary conditions at the metal surface using the images does not on its own produce the contact interaction between the image molecule and the electronic modes in the metal, which separately introduces the higher damping rate $\Gamma$. The method of images technique works well at low frequencies $\left(\nu \ll \omega_{\mathrm{p}}\right)$, with $\omega_{\mathrm{p}}$ being the plasma frequency, below which the metal is a good reflector. This well-established technique can account for the force of attraction between a charge and an uncharged dielectric medium, Van der Waals interactions between molecules and surfaces, quantum electrodynamical effects such as modified spontaneous emission rates ${ }^{18}$ and the Casimir-Polder force. ${ }^{19}$

To begin with we consider a single point charge, $q$ placed in a medium of relative permittivity, $\varepsilon_{1}$ at a distance, $d$ from a plane interface (defined by the $x-y$-plane) with a second medium of relative permittivity, $\varepsilon_{2}$. The field in medium 1 can be obtained if we replace the plane interface by an image charge with magnitude

$$
q^{\prime}=-\left(\frac{\varepsilon_{2}-\varepsilon_{1}}{\varepsilon_{2}+\varepsilon_{1}}\right) q
$$

at the image point in the surface of the first charge, which is at distance $2 d$ from it. The negative sign in eqn (1) arises because the field from $q^{\prime}$ now has opposite $x$ and $y$ components but the same $z$ component, so that $q^{\prime}$ is effectively a reflected image of $q$. A neutral electric dipole near such an interface with dipole moment, $\mu$ may be similarly described by removing the surface and replacing it with a second dipole at the image point by a dipole moment with magnitude (Fig. 1c)

$$
\mu^{\prime}=-\left(\frac{\varepsilon_{2}-\varepsilon_{1}}{\varepsilon_{2}+\varepsilon_{1}}\right) \tilde{\mu}
$$

where $\tilde{\mu}$ is the mirror image of the original dipole. In this way we can introduce an image of the real molecule situated inside the metal.
We require one additional feature before we can apply the method of images to SERS and this is to allow the dipole to oscillate with an angular frequency, $\omega$. If we write the time-dependent dipole as the real part of the complex dipole moment $\mu \mathrm{e}^{-i \omega t}$ then the associated complex image dipole will be

$$
\mu^{\prime} \mathrm{e}^{-i \omega t}=-\left(\frac{\varepsilon_{2}(\omega)-\varepsilon_{1}(\omega)}{\varepsilon_{2}(\omega)+\varepsilon_{1}(\omega)}\right) \tilde{\mu} \mathrm{e}^{-i \omega t}
$$

The two frequency-dependent permittivities are complex quantities; this applies in particular to $\varepsilon_{2}(\omega)$ because of resistive damping in the metal. Thus the image model of SERS comprises a molecular dipole and its image both coupled to an exponentially localized surface plasmon mode. ${ }^{20}$ This mode has a frequency at or very near to the surface plasmon resonance at which the real part of $\varepsilon_{2}(\omega)+\varepsilon_{1}(\omega)$ is very small and it follows that when the molecule is driven at this frequency a very large image dipole moment is also excited. It is the Raman emission from both the molecule and its image that produces the observed spectrum. In general there will be many Raman lines contributing to the SERS spectrum and these will depend on the vibrational modes specific to the molecule of interest as well as the nature of its environment. It is this very feature that underpins the utility of Raman spectroscopy. Here we do not focus on any particular molecule or treat the full spectrum but rather concentrate on the emission from a single vibrational Stokes line and its anti-Stokes counterpart. This greatly simplified system suffices for our purposes.

The probability amplitude for making a Raman transition from the electronic ground-state and vibrational state, $\nu$ to the electronic ground-state and vibrational state, $\nu^{\prime}$ is proportional to the molecular tensor,

$$
\alpha_{i j}^{0 \nu^{\prime}, 0 \nu}(\omega)=\left\langle\chi_{0 \nu^{\prime}}\left|\sum_{r}\left(\frac{\mu_{i}^{0 r} \mu_{j}^{r 0}}{E_{r 0}-\hbar \omega}+\frac{\mu_{j}^{0 r} \mu_{i}^{r 0}}{E_{r 0}+\hbar \omega}\right)\right| \chi_{0 \nu}\right\rangle
$$

where $\left\langle\chi_{0 \nu^{\prime}}\right|$ is the molecular electronic ground state with vibrational quantum number $\nu^{\prime}, E_{r 0}$ is the energy difference between the ground state and the excited state $r, \mu^{0 r}$ is the dipole matrix element between the ground state and $r$, while $i$ denotes the polarization of the emitted Raman $E$ field, $j$ is the polarization of the incident $E$ field and $\omega$ is the frequency of the exciting radiation.

A Raman transition in a molecule proceeds via the resonance excitation to a virtual excited state through the absorption of a photon of frequency $\omega$ from the pump laser. Then a Raman photon of frequency $\omega^{\prime}$ is emitted, so as to conserve energy, leaving the molecule in either its original vibrational ground state $\nu=0$ or in a different vibrational ground state $\nu^{\prime}$. If the state $\nu^{\prime}$ has a higher energy than the initial vibrational ground state $\nu$, then the emitted Raman photon will be of lower frequency than the laser frequency $\omega$ and a Stokes line is produced ( $\nu=0 \Rightarrow \nu^{\prime} \neq 0$ ) (Fig. 2a). If, however the state $\nu^{\prime}$ has a lower energy than the initial vibrational ground state $\nu$ then the emitted Raman photon will be of higher frequency than the laser frequency $\omega$ and an anti-Stokes line is produced $\left(\nu \neq 0 \Rightarrow \nu^{\prime}=0\right)$ (Fig. 2b). A typical SERS spectrum for both 

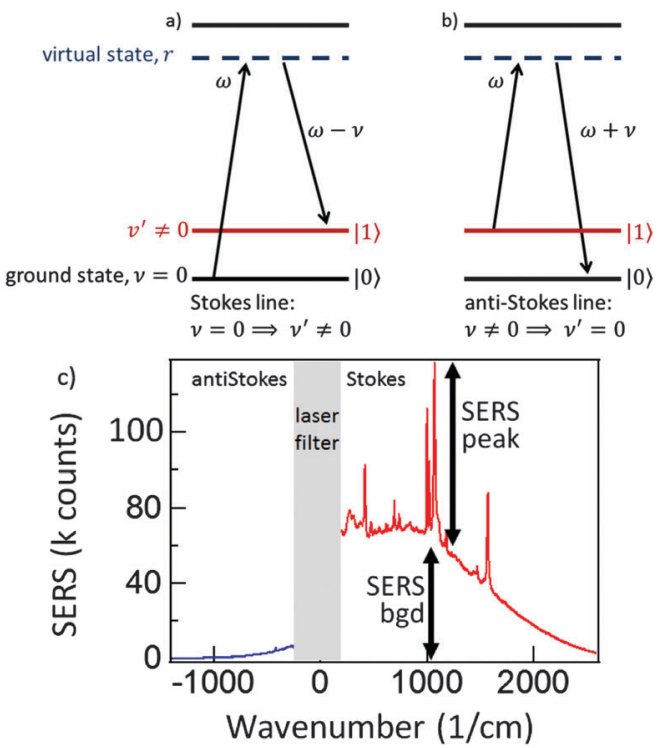

Fig. 2 (a) Stokes emission when the emitted Raman photon has a lower frequency than the pump laser $\omega$, and (b) anti-Stokes emission when the emitted Raman photon has a higher frequency than the pump laser (c) Experimental SERS spectrum from a monolayer of benzenethiol on Klarite $^{T M}$ showing typical SERS peaks and background for Stokes and antiStokes scattering.

Stokes and anti-Stokes scattering is seen in Fig. 2c, with similar enhancements for both the vibrational peaks, and the ever present background.

Both the real molecule and its image molecule contribute to this Raman transition process and, for the Stokes line, the result is the superposition of two Lorentzian functions which produce a line shape of the form

$$
S(\Omega)=\frac{\gamma_{\nu^{\prime}}}{\left(\Omega-\omega_{\nu^{\prime}}\right)^{2}+\pi^{2} \gamma_{\nu^{\prime}}{ }^{2}}+\left|\frac{\varepsilon_{2}(\omega)-\varepsilon_{1}(\omega)}{\varepsilon_{2}(\omega)+\varepsilon_{1}(\omega)}\right|^{4} \frac{\Gamma_{\nu^{\prime}}}{\left(\Omega-\omega_{\nu^{\prime}}\right)^{2}+\pi^{2} \Gamma_{\nu^{\prime}}{ }^{2}}
$$

where $\Omega$ is the frequency relative to that of the pump laser $\omega$, $\omega_{\nu^{\prime}}$ is the frequency of the vibrational state $\nu^{\prime}, \gamma$ and $\Gamma$ are the decay rates from the vibrational state to the vibrational ground state for the real and image molecules respectively, and $\hbar \omega_{\nu^{\prime}}$ is the energy of the vibrational state relative to the vibrational ground state (see ESI $\uparrow$ for a detailed derivation of (5)).

The first term in eqn (5) is the Lorentzian associated with the decay of the real molecule from the excited vibrational state $\nu^{\prime} \neq 0$ to the vibrational ground state $\nu=0$. Its characteristic line will be a sharp, narrow peak because typically the damping, $\gamma_{\nu^{\prime}}$ of the real molecule by the metal surface will be rather small; these are the normal SERS vibrational peaks. The second term is the Lorentzian associated with the decay of the image molecule from the excited vibrational state $\nu^{\prime} \neq 0$ to the vibrational ground state $\nu=0$. In this case however, the charges in the metal will tend to oscillate in sympathy with the image molecule producing heavy resistive damping, $\Gamma_{\nu^{\prime}}$ (associated with the imaginary part of the metal permittivity) until such time when the excited vibrational state will eventually decohere

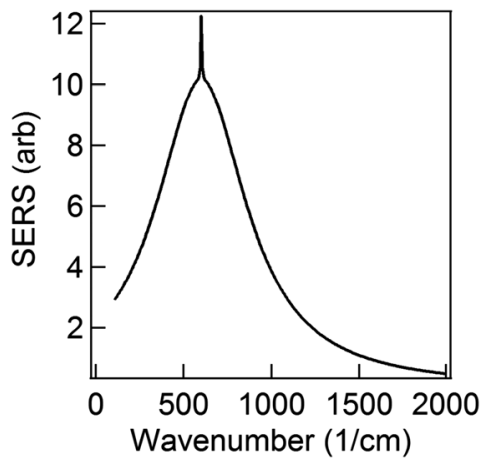

Fig. 3 Modeled spectrum Stokes spectrum of a broad background peak under a weaker and narrow Raman line, from (5) with $\operatorname{Re}\left\{\left|\frac{\varepsilon_{2}(\omega)-\varepsilon_{1}(\omega)}{\varepsilon_{2}(\omega)+\varepsilon_{1}(\omega)}\right|^{4}\right\}=10, \gamma_{\nu^{\prime}}=1 \mathrm{~cm}^{-1}$ and $\Gamma_{\nu^{\prime}}=100 \mathrm{~cm}^{-1}$.

into the vibrational ground state. This heavy damping means that $\Gamma_{\nu^{\prime}} \gg \gamma_{\nu^{\prime}}$ which will have the effect of producing a large broad continuum background, much broader than the first Lorentzian term giving the normal SERS peak. Crucially, the background continuum is only visible due to the enhancement factor $\left|\frac{\varepsilon_{2}(\omega)-\varepsilon_{1}(\omega)}{\varepsilon_{2}(\omega)+\varepsilon_{1}(\omega)}\right|^{4}$ associated with the large magnitude of the image dipole, $\mu^{\prime}$, oscillating at the surface-plasmon frequency. It is this factor that explains why the SERS background is only evident when there is interaction between both molecules and plasmons. ${ }^{16}$ The fourth power arises because the transition probability depends on the fourth power of the dipole moment. The modeled Stokes spectrum from eqn (5), depicting a narrow Raman line on a broad background, is shown in Fig. 3 based on conditions that give $\operatorname{Re}\left\{\left|\frac{\varepsilon_{2}(\omega)-\varepsilon_{1}(\omega)}{\varepsilon_{2}(\omega)+\varepsilon_{1}(\omega)}\right|^{4}\right\}=10, \gamma_{\nu^{\prime}}=1 \mathrm{~cm}^{-1}$ and $\Gamma_{\nu^{\prime}}=100 \mathrm{~cm}^{-1}$ (where $\operatorname{Re}\{\chi\}$ denotes the real part of $\chi$ ).

To examine the dependence of the Raman peak on $\gamma_{\nu^{\prime}}$ we fix all other variables constant (to the values of Fig. 3) and vary $\gamma_{\nu^{\prime}}$ from $0.01 \mathrm{~cm}^{-1}$ to $>1 \mathrm{~cm}^{-1}$. When $\gamma_{\nu^{\prime}}=0.01 \mathrm{~cm}^{-1}$ the Raman peak is initially very narrow, with a very large amplitude (due to $\gamma_{\nu^{\prime}}$ in the denominator of the first term in (5)) (Fig. 4a). This amplitude decreases as $\gamma_{\nu^{\prime}}$ is increased beyond $1 \mathrm{~cm}^{-1}$ (Fig. 4b), and disappears into the broad background when $\gamma_{\nu^{\prime}}=\Gamma_{\nu^{\prime}}=10$ $\mathrm{cm}^{-1}$ (Fig. 4c).

Similarly if all other variables are held constant and we vary $\Gamma_{\nu^{\prime}}$ from $1 \mathrm{~cm}^{-1}$ to $100 \mathrm{~cm}^{-1}$ we see that there is a very narrow peak, similar to the narrow Raman spectrum (but not in magnitude due to the $\left|\frac{\varepsilon_{2}(\omega)-\varepsilon_{1}(\omega)}{\varepsilon_{2}(\omega)+\varepsilon_{1}(\omega)}\right|^{4}$ term) when $\Gamma_{\nu^{\prime}}=\gamma_{\nu^{\prime}}=1$ $\mathrm{cm}^{-1}$ (Fig. 4d). The broad background starts to become evident as we increase the damping $\Gamma_{\nu^{\prime}}$ to values roughly $20 \times$ that of $\gamma_{\nu^{\prime}}$ $\left(\Gamma_{\nu^{\prime}}=20 \mathrm{~cm}^{-1}\right.$ ) (Fig. 4e). Both the narrow Raman peak and the broader background are fully evident in the Stokes spectrum when $\Gamma_{\nu^{\prime}}>40 \mathrm{~cm}^{-1}$ (Fig. 4f). The combination of the many different Lorentzian background components for each molecule leads to the overall background observed in experiment, 

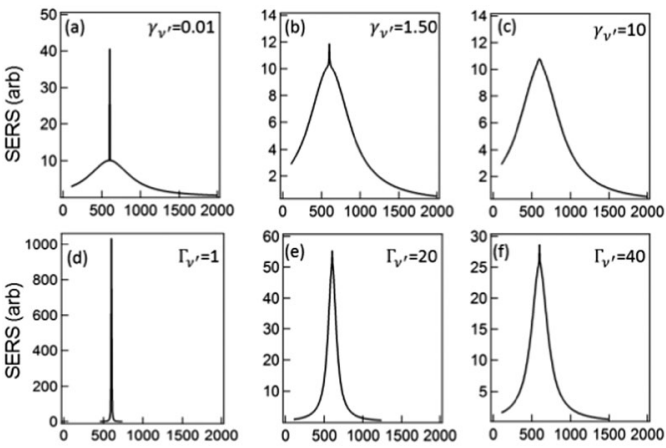

Wavenumber $(1 / \mathrm{cm})$

Wavenumber $(1 / \mathrm{cm})$

Fig. 4 Modeled Stokes spectra from eqn (5) with varying values of $\gamma_{\nu^{\prime}}$ and $\Gamma_{\nu^{\prime}}$ using $\operatorname{Re}\left\{\left|\frac{\varepsilon_{2}(\omega)-\varepsilon_{1}(\omega)}{\varepsilon_{2}(\omega)+\varepsilon_{1}(\omega)}\right|^{4}\right\}=10$ held constant, for $(\mathrm{a}-\mathrm{c}) \Gamma_{\nu^{\prime}}=$ $100 \mathrm{~cm}^{-1}$ and (a) $\gamma_{\nu^{\prime}}=0.01 \mathrm{~cm}^{-1}$, (b) $\gamma_{\nu^{\prime}}=1.5 \mathrm{~cm}^{-1}$, (c) $\gamma_{\nu^{\prime}}=10 \mathrm{~cm}^{-1}$. (d and e) $\gamma_{\nu^{\prime}}=1 \mathrm{~cm}^{-1}$ and (d) $\Gamma_{\nu^{\prime}}=1 \mathrm{~cm}^{-1}$, (e) $\Gamma_{\nu^{\prime}}=20 \mathrm{~cm}^{-1}$, (f) $\Gamma_{\nu^{\prime}}=40 \mathrm{~cm}^{-1}$.

with a resulting shape that reflects only the density of possible Raman transitions.

Let us now turn our attention to the anti-Stokes region of the spectrum. An anti-Stokes line is at a higher frequency than that of the pump laser, $\omega$ and corresponds to a transition from a higher to a lower vibrational state $\left(\nu \neq 0 \Rightarrow \nu^{\prime}=0\right)$ (Fig. 2b). At first sight we might expect both a narrow line corresponding to emission from the molecule and a background emitted by the image molecule. The fact that there is no broad background, however, is a further consequence of the same rapid resistive damping, $\Gamma_{\nu^{\prime}}$, that is responsible for the broad background on the Stokes side of the spectrum. A vibrational excitation in the image molecule dissipates very rapidly and hence Raman transitions occur only from the vibrational ground state $(\nu=0)$ for the image. Therefore energy conservation dictates that such transitions can give rise only to Stokes lines. ${ }^{16}$ This consideration thus produces a spectrum resembling that from experiment as seen in Fig. 2c.

We should ask if there is any interference between the Stokes emission of the molecule and the image molecule. To see there is none, we can appeal to quantum mechanics. After the emission of a Stokes photon we could, at least in principle, examine the real molecule to see if it is in the excited vibrational state, $\nu^{\prime}$. If it is then the emission must have come from the real molecule but if it is in its vibrational ground state, $(\nu=0)$ then the emission must have come from the image molecule. The existence of this "which-way" information suffices to tell us that there cannot be any interference in this case, which is indeed reproduced by the exact calculations.

Finally let us discuss the coupling, $V$ between the molecule and its image. The molecular dipole excited by the pump laser and its image molecule can couple to each other by means of the dipole-dipole interaction familiar from electrostatics. ${ }^{19}$ Let the metal surface define the $x-y$ plane so that the molecular dipole lies on the $z$ axis at a distance $\mathrm{d}$ from the surface as in Fig. 1c. The complex molecular and image dipoles are related

by eqn (3). The cycle-averaged potential energy associated with this interaction has the simple form

$$
V=\frac{1}{32 \pi \varepsilon_{0} d^{3}} \operatorname{Re}\left(2 \mu_{z} \mu_{z}^{\prime *}-\mu_{x} \mu_{x}^{\prime *}-\mu_{y} \mu_{y}^{\prime *}\right) .
$$

where $\varepsilon_{0}$ is the vacuum permittivity, and $\mu$ and $\mu^{\prime}$ are the real and image dipole components with respect to the surface in the $x-y$ plane. This coupling suggests the possibility of Förster-type energy transfer between the molecule and the image. ${ }^{21,22}$ The Raman transitions described above comprise of two steps. Virtual excitation of the molecule is immediately followed by de-excitation to a different vibrational level. The energy-transfer process proceeds instead in three steps. Firstly there is a virtual excitation of the molecule, which is followed by transfer of this excitation to the image molecule, mediated by the dipoledipole interaction, and finally the molecule relaxes to the ground state. Although this is a higher order process than the Raman transition, the strength of the image dipole excited at the pump frequency means that it may contribute significantly to the observed spectrum. The effect such a process has on the Stokes and anti-Stokes lines is as follows. The Stokes spectrum is made up of transitions from lower to higher vibrational levels (Fig. 2a). This means that the proposed energy-transfer process will leave the image molecule in a strongly damped excited vibrational state. Hence the net effect of including this process should be only a small correction to the large background. In the anti-Stokes spectrum the molecule is initially in an excited vibrational state and the energy-transfer process leaves the both the real and image molecules in their vibrational ground state, which is stable. The line-width for this process is determined, principally, by the lifetime of the initial vibrational state of the molecule. These initial and final states are the same as those for the lower-order Raman process and we therefore expect that there should be interference between these processes. If the interference is constructive this results in a stronger line but if it is destructive then we will get a partial cancellation. The strong distance dependence of the dipole-dipole coupling may lead to method to directly test this idea.

The ratio between the SERS peaks and background is thus set by a number of factors. Experimentally we find SERS peak linewidths are of order $\gamma=1 \mathrm{~cm}^{-1}$, which reduces as the molecules are further distanced from the surface towards the unperturbed Raman linewidths. Typical backgrounds observed have linewidths exceeding $\Gamma=100 \mathrm{~cm}^{-1}$, since the backgrounds from different vibrational lines are always blurred together. Moreover we assume here the simplest case in which the Raman selection rules are identical for real and image molecules, however this may not always be the case. For instance the rapid decay of plasmonic fields inside the metal can lead to a significant gradient in field strength along a normally Ramaninactive vibrational bond, and thus making background transitions visible without any large corresponding SERS peak from the real molecule. As the molecule is moved away from the surface, the strength of both SERS peaks and backgrounds will decrease as the local field exciting the dipoles reduces. However the ratio between these SERS contributions will be modified if 


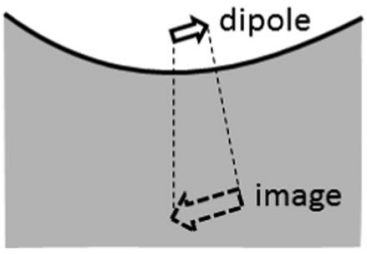

Fig. 5 Magnified image dipole for negative curvature roughness.

the metallic surface is not flat as assumed throughout here, but has some curvature on the scale of the separation involved. For instance a concave surface of negative radius of curvature $R$ leads to further magnification of the image dipole by a factor $[1-d / R]^{-3}$ (Fig. 5). This for instance can enhance the background contribution to SERS by $50 \%$ for only $R=10 \mathrm{~nm}$ scale roughness with molecules at $d=1.2 \mathrm{~nm}$ above the surface. We thus expect the ratio of peak to background SERS to be highly sensitive to the nanoscale morphology of the surfaces, which is indeed found experimentally, ${ }^{16}$ but needs further careful work to verify directly. However it suggests that to reduce the ratio of the SERS background for improving the signal to noise in SERS sensing, target molecules should be close to sharply convex nano-patterned surfaces, matching more recent observations on nanoparticles and etched nanostructures.

Various criticisms can be levelled at the simplicity of the model adopted here. Image charge models have a long history in SERS, and are known to possess various limitations. ${ }^{7,12,23}$ We indeed noted already that the distance dependence of molecule to surface of the SERS is not explicitly included (it scales with the plasmon field intensity at the molecule), but we are concerned here only with the ratio of background to SERS peaks. We explicitly postulated that the image and molecule have different damping to their environments, but this is the aspect that is normally left out of image charge models. However our model does agree with experiments that show the background observed does always scale with the SERS peak strength. Additional spectral variation in the background observed can arise from spectral filtering by the finite width of plasmonic resonance used to enhance the Raman, and this is only implicitly included here. The reason continuum backgrounds are not seen for molecules on completely flat surfaces is that in this case no external coupling is possible to the plasmons which are bound to the surface, and these are needed to provide the enhanced field used to get SERS, and to couple the scattering plasmons back into far field photons. Finally the image model is not suited to higher level precise descriptions of the SERS effects, as it does not include retardation sufficiently well, nor can it simply account for polarization selection rules. However the basic simplicity of the model to solve the long running question of the origin of the continuum background is strongly in its favour.

\section{Conclusions}

In summary we have shown that the method of images approach can be used to explain the spectra associated with a system of a vibrating SERS molecule interacting with a resonant, plasmonic metal surface. By replacing the molecular-surface interaction with a molecular-image interaction we derive an equation for the Stokes line combining two Lorentzian terms. The first Lorentzian term produces a narrow sharp peak associated with a normal SERS line, due to the relatively small amount of damping between the molecule and the plasmonic surface. The second Lorentzian term produces a large continuum spectrum, referred to as the SERS background, from the dissipative, resistive damping of the image molecule due to the imaginary part of the metal refractive index. Such an intuitive model is of crucial importance in developing spectroscopies with improved signal to noise (not just enhanced signal) as needed for ultra-sensitive sensing.

We gratefully acknowledge data and stimulating discussions provided by Sumeet Mahajan and Phil Bartlett. This work was supported by UK EPSRC EP/C511786/1, EP/F011393, ERC 320503 LINASS, EU CUBIHOLES, 3M, the Royal Society and the Wolfson Foundation.

\section{Notes and references}

1 K. A. Willets and R. P. Van Duyne, Annu. Rev. Phys. Chem., 2007, 58, 267-297.

2 X. Qian, X.-H. Peng, D. O. Ansari, Q. Yin-Goen, G. Z. Chen, D. M. Shin, L. Yang, A. N. Young, M. D. Wang and S. Nie, Nat. Biotechnol., 2008, 26, 83-90.

3 K. Kneipp, Y. Wang, H. Kneipp, L. Perelman, I. Itzkan, R. Dasari and M. Feld, Phys. Rev. Lett., 1997, 78, 1667-1670.

4 M. D. Porter, R. J. Lipert, L. M. Siperko, G. Wang and R. Narayanan, Chem. Soc. Rev., 2008, 37, 1001-1011.

5 D. A. Stuart, A. J. Haes, C. R. Yonzon, E. M. Hicks and R. P. Duyne, Van IEEE Proc.-Nanobiotech., 2005, 152, 13-32.

6 R. M. Amos and W. L. Barnes, Phys. Rev. B: Condens. Matter Mater. Phys., 1997, 55, 7249-7254.

7 M. Moskovits, J. Raman Spectrosc., 2005, 36, 485-496; M. Moskovits, Rev. Mod. Phys., 1985, 57, 783-826.

8 K. D. Osberg, M. Rycenga, N. Harris, A. L. Schmucker, M. R. Langille, G. C. Schatz and C. A. Mirkin, Nano Lett., 2012, 12, 3828-3832.

9 A. Bagchi, R. G. Barrera and B. B. Dasgupta, Phys. Rev. Lett., 1980, 44, 1475-1478.

10 E. Burstein, Y. J. Chen, S. Lundquist and E. Tosatti, Solid State Commun., 1979, 29, 567-570.

11 J. I. Gersten, R. L. Birke and J. B. Lombardi, Phys. Rev. Lett., 1979, 43, 147-150.

12 W. H. Weber and G. W. Ford, Phys. Rev. Lett., 1980, 4, 1774-1777.

13 A. Campion, P. Kambhampati and C. Harris, Chem. Soc. Rev., 1998, 27, 241-250.

14 D. Cunningham, R. E. Littleford, W. E. Smith, P. J. Lundahl, I. Khan, D. W. McComb, D. Graham and N. Laforest, Faraday Discuss., 2006, 132, 135-145.

15 T. E. Furtak and J. Reyes, Solid State Commun., 1980, 93, 351-382. 
16 S. Mahajan, R. M. Cole, J. D. Speed, S. H. Pelfrey, A. E. Russell, P. N. Bartlett, S. M. Barnett and J. J. Baumberg, J. Phys. Chem. C, 2010, 114, 7242-7250.

17 J. D. Jackson, Classical Electrodynamics, John Wiley \& Sons, Inc., 3rd edn, 1998.

18 P. W. Milonni and P. L. Knight, Opt. Commun., 1973, 9, 119-122.

19 S. M. Barnett, A. Aspect and P. W. Milonni, J. Phys. B: At., Mol. Opt. Phys., 2000, 33, L143-L149.
20 O. Madelung, Introduction to Solid State Theory, Springer, Berlin, 1996.

21 T. Forster, Modern Quantum Chemistry, Academic Press, New York, 1965, pp. 93-137.

22 P. Wu and L. Brand, Anal. Biochem., 1994, 218, $1-13$.

23 F. W. King, R. P. Van Duyne and G. C. Schatz, J. Chem. Phys., 1978, 69, 4472. 